Available online at www.eccomasproceedia.org

Eccomas Proceedia COMPDYN (2021) 2791-2799

ECCOMAS

Proceedia
COMPDYN 2021

$8^{\text {th }}$ ECCOMAS Thematic Conference on Computational Methods in Structural Dynamics and Earthquake Engineering M. Papadrakakis, M. Fragiadakis (eds.) Streamed from Athens, Greece, 28 - 30 June 2021

\title{
EXPERIMENTAL IDENTIFICATION OF MECHANICAL AND DYNAMIC CHARACTERISTICS OF 3D PRINTING SPECIMENS
}

\author{
Ioannis E. Kavvadias ${ }^{1}$, Konstantinos Tsongas ${ }^{2}$, Dimitrios Tzetzis², Kosmas E. Bantilas ${ }^{1}$, \\ Lazaros Vasiliadis ${ }^{1}$, Anaxagoras Elenas ${ }^{1}$ \\ ${ }^{1}$ Department of Civil Engineering, Democritus University of Thrace \\ Campus of Kimmeria, Xanthi 67100, Greece \\ e-mail: \{ikavvadi, kbantila, lvasilia,elenas\}@civil.duth.gr \\ ${ }^{2}$ Digital Manufacturing and Materials Characterization Laboratory, School of Science and Technology \\ International Hellenic University \\ 14km Thessaloniki - N. Moudania, Thermi GR57001, Greece \\ \{k.tsongas, d.tzetzis\}@ihu.edu.gr
}

\begin{abstract}
Additive manufacturing is at the forefront of research activities worldwide, as the commercial exploitation of this technology is expected to displace some traditional manufacturing methods over the next few years. The aim of this research is the identification of the dynamic characteristics of three-dimensional printing specimens. The specific specimens, composed of $3 D$ printed parts, correspond to elastic single and multiple degree of freedom oscillators. Moreover, parts that represent rigid rocking columns are constructed. Bending tests are performed in order to identify the stiffness of the elastic oscillators. Moreover, experimental results of free vibrations are presented in order to investigate the dynamic response of the rocking parts. The dynamic behavior verification through analytical solutions will highlight the adequacy of $3 D$ printing specimens for conducting experimental research in structural dynamics.
\end{abstract}

Keywords: Additive Manufacturing, Fused Deposition Modeling, Structural Dynamics, Earthquake Engineering, Rocking Structures, Experimental Investigation. 


\section{INTRODUCTION}

Additive manufacturing technology is widely applied to optimize products in several industries and more specifically in the field of civil engineering [1] where the efforts have been intensified recently towards the implementation and testing of protypes. Additive Manufacturing (AM), also known as 3D Printing, allows the fabrication of fully personalized designs with geometrical complexity, while it decreases the use of tools, the cost during prototyping steps and the fabrication time [2]. No requirement of molds, costly tools, milling, and sanding processes are the main reasons why it is a low-cost production method. Even though the basic methods of thermoplastics forming (extrusion, injection molding, thermoforming) are the mainstream in the polymers industry, 3D printing is more efficient, time saving, and minimizes the use of raw materials [3]. During the past few decades, different applications of 3D printing have been investigated in various industries, due to the interest of scientists, engineers, and the medical community [4]. The extensive use of 3D printing in recent years has increased the interest in Fused Deposition Modelling (FDM), which is a widely used method for 3D printing, due to good efficiency, easy material deposition and low costs [5]. Over the last years, bio-based polymers, such as poly (lactide acid) (PLA), have attracted much attention in 3D printing, replacing petroleum-based polymers, due to its availability from renewable and environmentally friendly resources as well its outstanding properties as high tensile strength and modulus $[6,7]$, demonstrating simultaneously high-stiffness and high-damping properties, worthy of further functional studies [8]. The application of PLA in FDM technology raises year by year [9]. The mechanical response of 3D printed PLA was found better than injection-molded PLA [10]. However, PLA has some disadvantages, such as poor thermal stability and surface, as well as brittleness which make it unsuitable for some large-scale end uses.

In the present research project, the exploitation of 3D printing in seismic testing was investigated. Especially, the seismic response of rocking podium strictures (RPSs) [11-13] was experimentally investigated. RPSs are comprised of an elastic superstructure placed on the top of a rocking story. Thus, 3D printed parts representing rocking columns and elastic oscillators were constructed. Evaluating the mechanical and the dynamic properties of the specimens by comparing the behavior with the analytical solution results, significant remarks arise regarding the capability of 3D printing in earthquake engineering experimental research. In the current study, preliminary results obtained by bending tests of the elastic members, as well as free rocking oscillation tests of rocking columns and frames are presented and discussed.

\section{ROCKING PODIUM STRUCTURES}

The first detailed study of the dynamic response of structures seismically isolated using a purely rocking floor at the base, considering that the superstructure behaves like a single degree of freedom elastic oscillator, was carried out by Bachmann et al. [11]. Based on the sdof model of the superstructure, Bantilas et al. [12] investigated the parameters that affect the elastic demands of RPSs. Later studies of Bantilas et al. [13] highlighted the critical effect of the higher vibration modes on the dynamic response of RPSs consisted of multiple degree of freedom (mdof) elastic superstructure. Experimental investigation of RPSs consisted of sdof superstructure has been also conducted [14]. The presence of curved extensions at the base of the rocking columns results in rolling and rocking rocking response [15]. The idealization of the model of mdof elastic oscillator fixed on the top of a rocking frame which is comprised of kinematic bearings is illustrated in Figure 1(a). Moreover, a typical configuration of the kinematic bearings is presented in Figure 1(b). 
The rigid frame consists of a cap beam with mass $\mathrm{m}_{\mathrm{b}}$ and $\mathrm{N}$ freestanding columns with mass $\mathrm{m}_{\mathrm{c}}$, semi-diagonal length $\mathrm{R}=\sqrt{\mathrm{H}^{2}+\mathrm{B}^{2}}$, rotational moment of inertia around its center of mass $\mathrm{I}_{\mathrm{cm}}$, and slenderness $\alpha=\operatorname{atan}(\mathrm{B} / \mathrm{H})$. In case of kinematic bearings with curved wedges and total slenderness $\tan \alpha^{\prime}=\mathrm{B}^{\prime} / \mathrm{H}$, the post uplift stiffness during rolling response depends on the radius of curvature $r$ of the extensions, while the wedge's angle $\beta$ sets the limit between rolling and rocking response. The superstructure is determined by the mass matrix $\mathbf{M}$, the stiffness matrix $\mathbf{K}$ and the damping matrix $\mathbf{C}$. The equations of motion of the examined structural system are presented in the aforementioned literature.
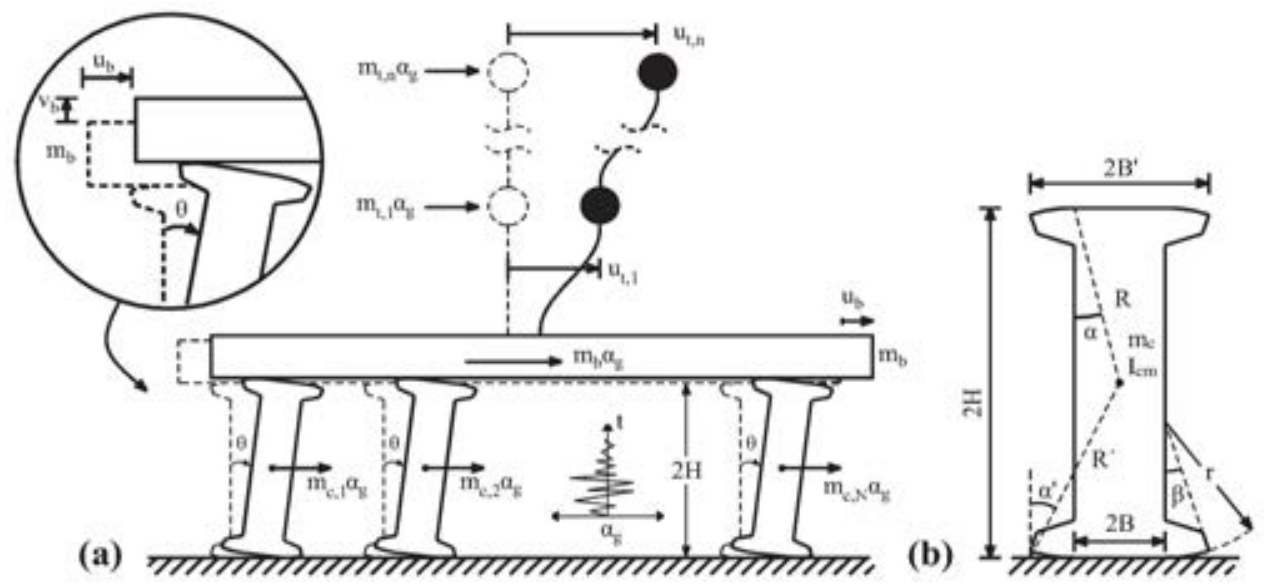

Figure 1: (a) Schematic representation of the analytical model, (b) geometric configuration of a typical kinematic bearing.

For the scope of the experimental investigation, rocking podium structures comprised by a couple of kinematic bearings, and superstructures which were formed by stiff plates and two flexible walls were designed. In total, three different sets of kinematic bearings were constructed. Moreover, a one storey superstructure, a three storey superstructure and a rigid superstructure were configured. In order to obtained comparable response results, the total mass of the superstructures is equal, while the flexible superstructures demonstrate the same fundamental vibration period.

Each rocking column has height $2 \mathrm{H}=150 \mathrm{~mm}$ and slenderness $\alpha=0.15 \mathrm{rad}$. Thus, the width was $2 \mathrm{~B}=22.5 \mathrm{~mm}$. Regarding the kinematic bearings with the curved extensions, the total width was $2 \mathrm{~B}^{\prime}=45 \mathrm{~mm}$ and the wedges' radius of curvature $r=50$ and $75 \mathrm{~mm}$. The rocking parts were placed on the top of a stiff plate.

The dynamic properties of the elastic oscillators should be in accordance with the scale of the rocking parts. Thus, taking into account that the height of the rocking parts is scaled down by a factor of 20 , the period of the elastic oscillators must be scaled down by in order to represent a full size podium structure. Assuming that a typical three storey structure demonstrate period $\mathrm{T}_{\mathrm{t}}=0.3 \mathrm{~s}$, the period of the specimens should be $\mathrm{T}_{\mathrm{t}}=0.07 \mathrm{~s}$. The elastic oscillators are comprised of stiff plates and flexural walls. The stiff plates have dimensions $150 \times 150 \times 6 \mathrm{~mm}$.

The vertical parts have height and width equal to $150 \mathrm{~mm}$. The most important dimension, which is defined by the targeted period, is the thickness of the walls. Considering that in typical structures the distribution of floor masses is constant, the translational stiffness distribution throughout the structure affects the lateral displacement profile and the effective mass of the fundamental vibration mode. Due to the assembly of the superstructure the plates should be considered as rigid, while the walls as flexible. As such, only translational Dofs were considered. The relative translational stiffness between the floors was determined by solving the eigenvalue problem [17]. Regarding the mdof superstructure, the eigenvalue problem was solved in reverse 
assuming triangular lateral displacement profile [16]. As such, given that the Youngs modulus of PLA is E $=3.2 \mathrm{GPa}$, the thickness of the walls of the sdof superstructure was $4.85 \mathrm{~mm}$, while for the mdof superstructure the thickness were $6.35 \mathrm{~mm}, 5.83 \mathrm{~mm}$ and $4.80 \mathrm{~mm}$ for the walls of the 1 st, the 2 nd and the 3 rd storey respectively.

The individual structural members of the examined structural system were designed in Solidworks CAD software (Dassault Systèmes, SolidWorks Corporation, Waltham, MA, USA). Except of the geometric characteristics referred above, of great importance was the detailed design of the regions where the assembly of the kinematic bearings with the base plate and the cup beam, and the plates with the flexible walls took place. In Figure 2 indicative structural members, as well as the details of the plates, as they are designed, are illustrated.
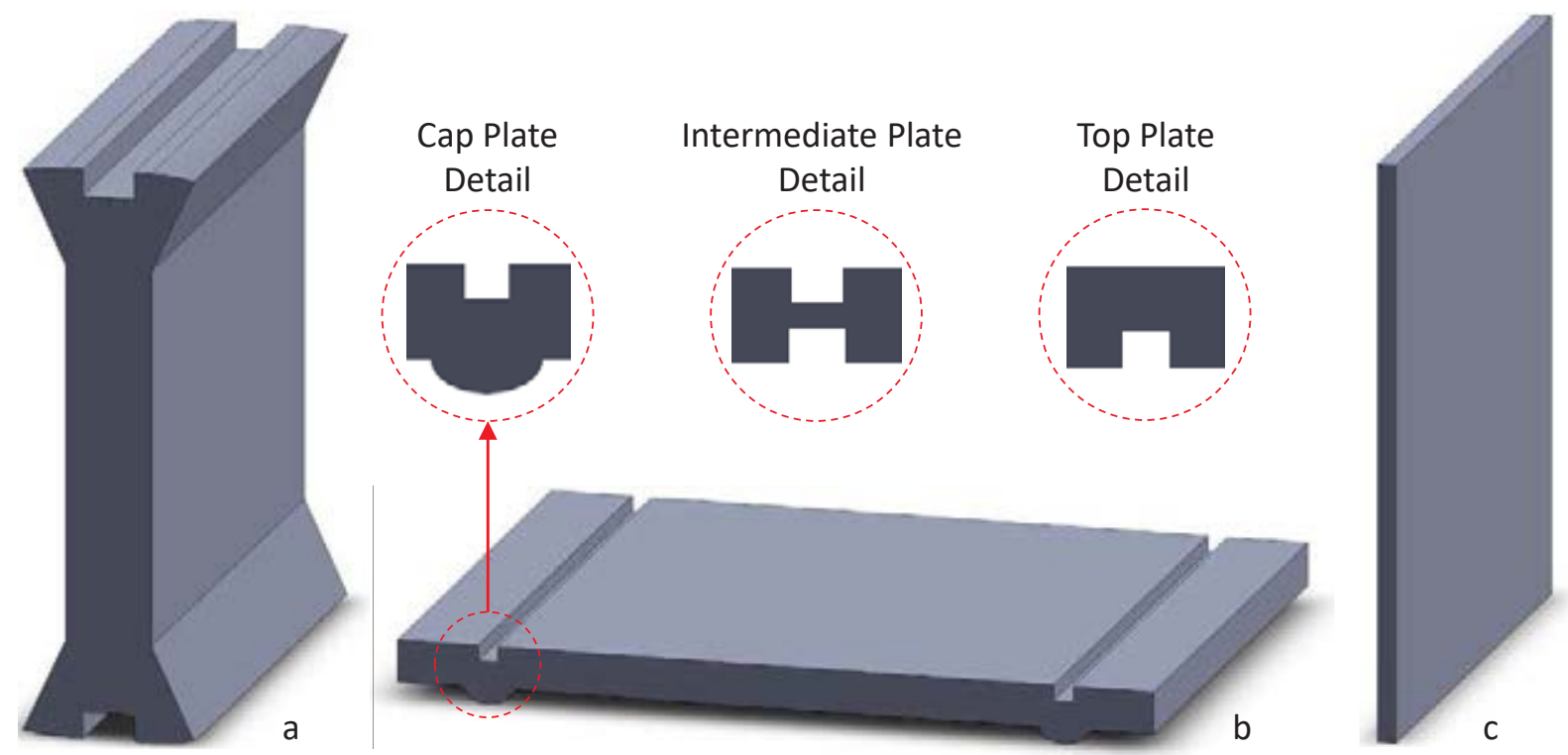

Figure 2: CAD models of (a) a kinematic bearing, (b) a plate and (c) an elastic wall.

\section{EXPERIMENTAL METHODS}

\subsection{Fabrication of 3D printed specimens}

All specimens tested in this study were produced on a BCN3D Sigma R17 3D printer (BCN3D, Barcelona, Spain) with a $2.85 \mathrm{~mm}$ filament diameter. BCN3D Cura was used as slicing Software. Only system integrated (default) variations of production parameters were used in the comparative analysis. The printing parameters used were: nozzle extrusion temperature of $220^{\circ} \mathrm{C}$, heat bed temperature of $60^{\circ} \mathrm{C}$, deposition line (layer) height $0.3 \mathrm{~mm}$, deposition line width $0.8 \mathrm{~mm}$ and printing speed of $60 \mathrm{~mm} / \mathrm{s}$. Deposition speed was therefore not considered a variable in this study and a constant extrusion velocity was selected for all specimens, based on device parameters (e.g., effective printing range). Also, 3D printing was performed in a standard laboratory without temperature or humidity control.

The kinematic bearing specimens have been $3 \mathrm{D}$ printed with an infill density of $20 \%$, as illustrated in Figure 3a. For the plates, an infill density of 30\% was considered optimal (Figure $3 b)$. Figure $3 \mathrm{c}$ demonstrates the elastic wall specimens that were $3 \mathrm{D}$ printed as hollow sections. For all specimens two lines were printed on the perimeter to form the outer shell, which is typical in FDM prints. Figure 3d shows an assembly of a rocking podium structure consisted of a three storey superstructure. It has to be mentioned that in order to avoid sliding, a low friction tape has been applied over the contact region between the bearings and the plates. 


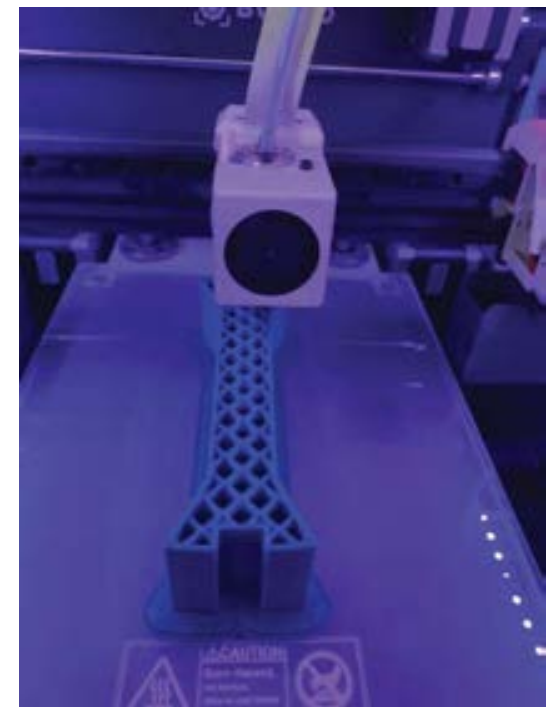

(a)

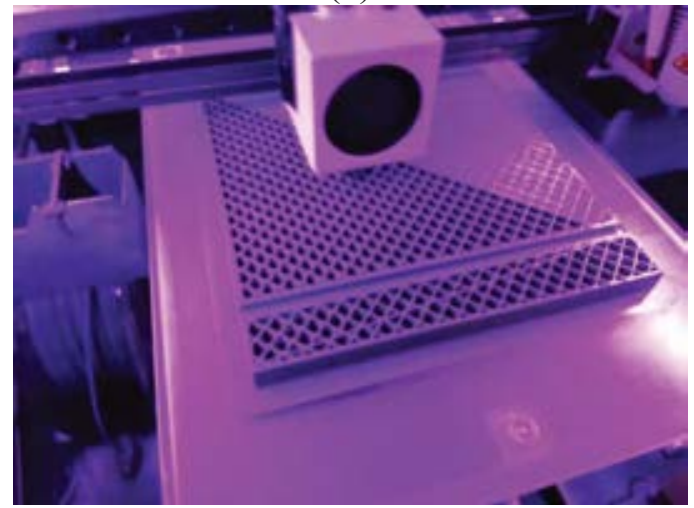

(b)

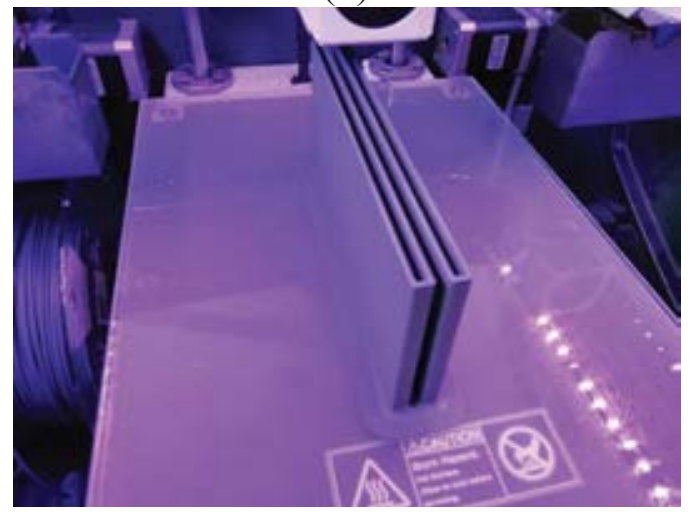

(c)

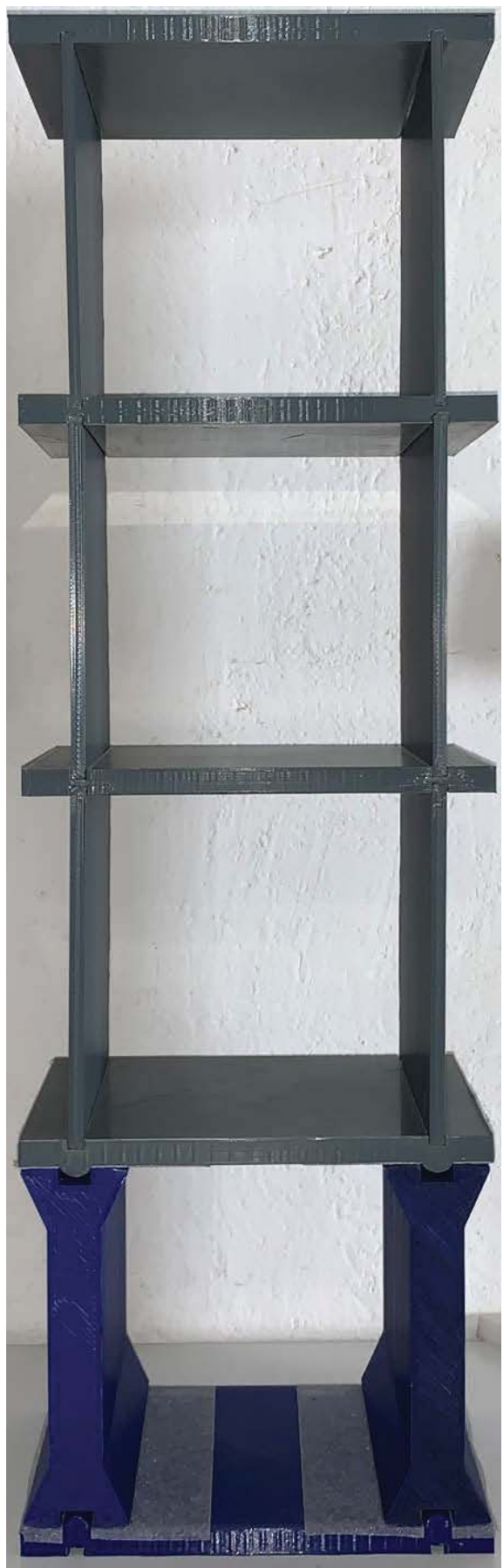

(d)

Figure 3: 3D printed (a) kinematic bearing, (b) plate, (c) elastic wall and (d) assembly of a rocking podium structure.

\subsection{Bending tests}

The 3D printed wall structures were subjected under 3-point bending tests using a universal Testometric M500-50AT testing machine equipped with $50 \mathrm{kN}$ load cell, according ASTM D590. The support span was set to be $110 \mathrm{~mm}$. At least three specimens were prepared and 
tested. The loading and supports noses have a diameter of $10 \mathrm{~mm}$. The crosshead speed was set to be $5 \mathrm{~mm} / \mathrm{min}$.

\subsection{Free oscillation tests}

The kinematic response of a kinematic bearing and a rocking frame, under free oscillation, was measured by motion tracking analysis of image sequences. For this scope the dynamic behavior is captured by a Canon EOS 5D MKIV camera with a Canon EF 24-70mm f/2.8 L USM II lens. The measurement of the displacements was performed using the Kinovea [18] software. Kinovea is a free and open source 2D motion tracking software that analyzes video files, even without markers. However, in order to improve the reliability of the measurements passive markers were assigned on the specimens.

\section{MECHANICAL AND KINEMATIC BEHAVIOR OF 3D PRINTED SPECIMENS}

Preliminary investigation of the identification of mechanical and kinematic properties of 3D printed specimens has been performed. These 3D printed parts will be used to construct the examined RPSs for the purpose of the current research program.

Orientation can also have a significant impact on print time. The 3D printing direction was purposely selected. For functional parts, it is important to consider the application and the direction of the loads. FDM parts are much more likely to delaminate and fracture when placed in tension in the $\mathrm{Z}$ direction compared to the $\mathrm{XY}$ directions.

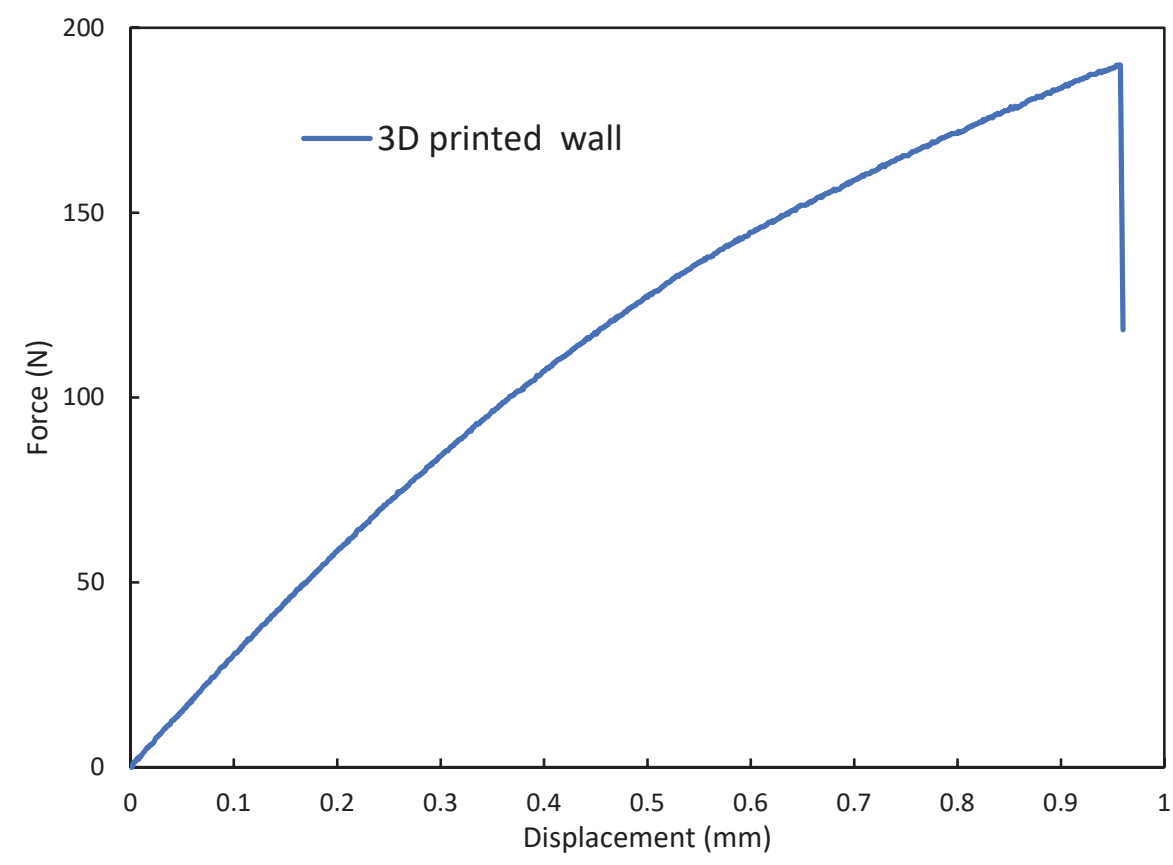

Figure 4: Typical force-displacement curve of a 3D printed elastic wall.

The force-displacement response of a 3D printed elastic wall has been determined with flexural tests. The typical force-displacement curve is illustrated in Figure 4. It can be observed that the maximum force that was applied on the 3D printed elastic wall was $205 \mathrm{~N}$. The deflection at break was $0.95 \mathrm{~mm}$. The flexural stiffness is a criterion of measuring deformability. The flexural stiffness of a structure is a function based upon two essential properties: the elastic modulus, E (stress per unit strain) of the material that composes it, and the moment of inertia, 
I, a function of the cross-sectional geometry. In terms of flexural stiffness EI, the mean measured value was $8.5 \times 10^{6} \mathrm{Nmm}^{2}$, while the theoretical calculations during the design process were given at $9 \times 10^{6} \mathrm{Nmm}^{2}$.

In Figure 5 the experimental time histories of an individual kinematic bearing and a rocking frame under free oscillation are presented comparatively with the analytical solution. Regarding the kinematic bearing with radius of curvature $r=50 \mathrm{~mm}$, the responses are demonstrated in terms of horizontal displacement of it center of mass (Figure 5a). The analytical response was calculated given the design properties of the bearing, while the coefficient of restitution that defines the energy dissipation during impact was assumed as provided by Bachmann et al. [15]. Given the geometry of the kinematic bearing and the printing properties, the non-dimensional moment of inertia $\overline{\mathrm{I}}_{0}=1.354$. Where $\overline{\mathrm{I}}_{\mathrm{o}}=\mathrm{I}_{\mathrm{o}} /\left(\mathrm{m}_{\mathrm{c}} \mathrm{R}^{2}\right)$, and $\mathrm{I}_{0}$ is the rotational inertia of the column with respect to the pivot point. In Figure $5 \mathrm{~b}$ the response of a rocking frame consisted of the kinematic bearings with radius of curvature $r=50 \mathrm{~mm}$ is displayed. The analytical solution was derived by Makris and Vassiliou [19]. The time history is given in terms of the cap beams' horizontal displacement. In general, the analytical solution predicts well enough the response time histories. Furthermore, the coefficient of restitution seems to provide with high precise the energy dissipation during impact. However, the differences between the responses, especially in case of the kinematic bearings, lead to significant remarks. It has to be mentioned the fact that although the amplitude of the negative values of the responses are in good agreement, the measured positive displacements are higher than those of the analytical solution. Moreover, the peak positive value after impact is almost equal to the negative extreme value before impact. These remarks imply a non-symmetric specimen. Although the initial design was totally symmetric, the eccentricity may occur due to imperfections of the external geometry or due to nonsymmetric pattern of the infill. In order to capture the response of the specimens with higher accuracy, validation of the analytical solution in needed.
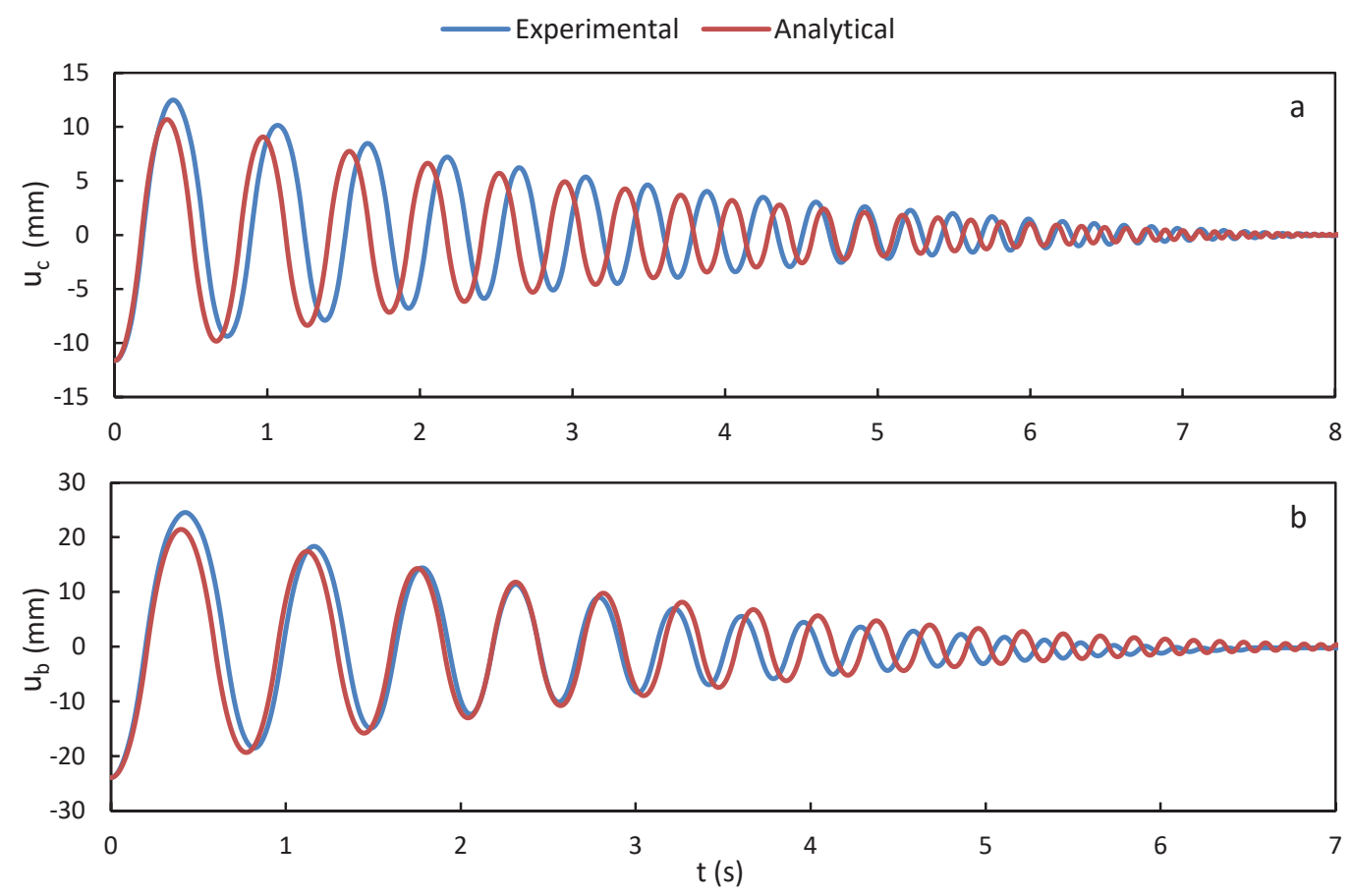

Figure 5: Response under free oscillation of (a) a kinematic bearing and (b) a rocking frame. 


\section{CONCLUSIONS}

In the present research project, the exploitation of 3D printing in seismic testing is investigated. 3D printed parts representing rocking columns and elastic oscillators were manufactured with Fused Deposition Modeling technology. The mechanical and the dynamic properties were assessed. The measured mechanical response was very close to the designed. Considering the dynamic behavior, minor deviations to the designed geometry were observed. Therefore, the $3 \mathrm{D}$ printing procedure can be potentially utilized for seismic testing. In a future study, the construction of a redesigned and more complex structural system could be done, through the process of additive manufacturing, along with a thorough examination of the dynamic behavior via the utility of proper experiments.

\section{ACKNOWLEDGEMENTS}

This research is co-financed by Greece and the European Union (European Social FundESF) through the Operational Programme «Human Resources Development, Education and Lifelong Learning» in the context of the project "Reinforcement of Postdoctoral Researchers 2nd Cycle" (MIS-5033021), implemented by the State Scholarships Foundation (IKY).

\section{REFERENCES}

[1] L. Del Giudice, M. F. Vassiliou, Mechanical properties of 3D printed material with binder jet technology and potential applications of additive manufacturing in seismic testing of structures. Additive Manufacturing, 36, 101714, 2020.

[2] C.M. González-Henríquez, M.A. Sarabia-Vallejos, J. Rodriguez-Hernandez, Polymers for additive manufacturing and 4D-printing: Materials, methodologies, and biomedical applications. Progress in Polymer Science, 94, 57-116, 2019.

[3] K. Gnanasekaran, T. Heijmans, S. Van Bennekom, H. Woldhuis, S. Wijnia, G. de With, H. Friedrich, 3D printing of CNT-and graphene-based conductive polymer nanocomposites by fused deposition modeling. Applied materials today, 9, 21-28, 2017.

[4] C. Wang, W. Huang, Y. Zhou, L. He, Z. He, Z. Chen, X. He, S. Tian, J. Liao, B. Lu, Y. Wei, M. Wang, 3D printing of bone tissue engineering scaffolds. Bioactive materials, 5(1), 82-91, 2020.

[5] P. Szymczyk-Ziółkowska, M.B. Łabowska, J. Detyna, I. Michalak, P. Gruber, A review of fabrication polymer scaffolds for biomedical applications using additive manufacturing techniques. Biocybernetics and Biomedical Engineering, 40(2), 624-638, 2020.

[6] D. Rasselet, A.S. Caro-Bretelle, A. Taguet, J.M. Lopez-Cuesta, Reactive compatibilization of PLA/PA11 blends and their application in additive manufacturing. Materials, 12(3), 485, 2019.

[7] W.J. Choi, K.S. Hwang, H.J. Kwon, C. Lee, C.H. Kim, T.H. Kim, S.W. Heo, J.H. Kim, J.Y. Lee, Rapid development of dual porous poly (lactic acid) foam using fused deposition modeling (FDM) 3D printing for medical scaffold application. Materials Science and Engineering: C, 110, 110693, 2020.

[8] M. Mansour, K. Tsongas, D. Tzetzis, Measurement of the mechanical and dynamic properties of 3D printed polylactic acid reinforced with graphene. Polymer-Plastics Technology and Materials, 58(11), 1234-1244, 2019. 
[9] V. Mazzanti, L. Malagutti, F. Mollica, FDM 3D printing of polymers containing natural fillers: A review of their mechanical properties. Polymers, 11(7), 1094, 2019.

[10] Y. Song, Y. Li, W. Song, K. Yee, K.Y. Lee, V.L. Tagarielli, Measurements of the mechanical response of unidirectional 3D-printed PLA. Materials and Design, 123, 154-164, 2017.

[11] J.A. Bachmann, M.F. Vassiliou, B. Stojadinović, Dynamics of rocking podium structures. Earthquake Engineering and Structural Dynamics, 46(14), 2499-2517, 2017.

[12] K.E. Bantilas, I.E. Kavvadias, L.K. Vasiliadis, Analytical investigation of the seismic response of elastic oscillators placed on the top of rocking storey. Bulletin of Earthquake Engineering, 19, 1249-1270, 2021.

[13] K.E. Bantilas, I.E. Kavvadias, L.K. Vasiliadis, Seismic response of elastic multidegree of freedom oscillators placed on the top of rocking storey. Earthquake Engineering and Structural Dynamics, 50(5), 1315-1333, 2021.

[14] Bachmann J.A., Jost C., Studemann Q., Vassiliou M.F., Stojadinović B., An analytical model for the dynamic response of an elastic SDOF system fixed on top of a rocking single-storey frame structure: experimental validation. 7th European Congress on Computational Methods in Applied Sciences and Engineering, Crete Island, Greece, 2016.

[15] J.A. Bachmann, M.F. Vassiliou, B. Stojadinović, Rolling and rocking of rigid uplifting structures. Earthquake Engineering and Structural Dynamics, 48(14), 1556-1574, 2019.

[16] G.E. Thermou, S.J. Pantazopoulou, A.S. Elnashai, Global interventions for seismic upgrading of substandard RC buildings. Journal of Structural Engineering, 138(3), 387-401, 2012.

[17] A.K. Chopra, Dynamics of Structures: Theory and Applications to Earthquake Engineering. Prentice Hall, 1995.

[18] https://www.kinovea.org/

[19] N. Makris, M.F. Vassiliou, Planar rocking response and stability analysis of an array of free-standing columns capped with a freely supported rigid beam. Earthquake Engineering and Structural Dynamics, 42(3), 431-449, 2013. 